\title{
MORPHOLOGICAL PROPERTIES OF UNUSUALLY WARM IRAS GALAXIES
}

\author{
Charlene Anne Heisler 1),2) and J. Patricia Vader 1) \\ 1) Dept. of Astronomy, Yale University, Box 6666, New Haven, CT., 06511 \\ 2) Guest Investigator, NOAO, Cerro Tololo Inter-American Observatory, Operated by \\ Associated Universities for Research in Astronomy, Inc.
}

A sample of unusually warm IRAS galaxies with spectral energy distributions peaking at $60 \mu \mathrm{m}$ is studied. The complete sample consisting of 21 known galaxies and 40 previously unidentified galaxies is selected according to the following criteria: all galaxies in IRAS Point Source Catalogue with high quality detections at $25 \mu \mathrm{m}$ and $60 \mu \mathrm{m}$, FIR fluxes such that $4>\mathrm{f}_{60} / \mathrm{f}_{25}>1$ and $\mathrm{f}_{60} / \mathrm{f}_{100}>1$, and a galactic latitude of $|\mathrm{b}|>10^{\circ}$. The colour ctireria select against galaxies with a significant cool dust component and the hotter dust present is presumably circumnuclear (Vader et al., 1988). CCD imaging reveals a morphological dichotomy in our sample - the galaxies are either relaxed systems (E/S0) or interacting (double nuclei, tidal features etc.). The relatively fast process of merging may link these two types of systems.

Only morphologically relaxed systems are considered here. An unsharp masking (Schweizer and Ford, 1985), and a two-colour (Sparks et al., 1985) technique were used to search for low surface brightness details and for dust. Of the 11 galaxies examined, 5 show features and 6 have no apparent fine structure (Table 1). An unsharp masked R frame and a B-R frame contour plot of 10567 (Fig. 1) reveal an extension with 3 red, presumably dusty, patches. This object has an unusually large far-infrared excess. An unsharp masked B frame contour plot, and a B-I colour frame of 14164 reveal 3 blue central knots (Fig. 2). (In both figures east is to the top and north is to the left of the page.)

This preliminary study shows that at least $50 \%$ of the morphologically relaxed galaxies in our sample have low surface brightness features (shells, dust) that may be a clue to the nature of their far-infrared emission.

TABLE 1

\begin{tabular}{lccll}
\hline OBJECT & TYPE & $\log \left(\mathrm{L}_{60} / \mathrm{L}_{\mathrm{B}}\right)$ & $\mathrm{m}_{\mathrm{B}}$ & FEATURES \\
$05570-8123$ & Sy 2 & 0.96 & 16.7 & dust lane \\
$09497-0122^{1}$ & Sy 1 & 0.48 & 15.3 & no apparent features \\
$10567-3323$ & HII & 1.70 & 17.9 & disturbed, 3 red patches \\
$13329-3402^{2}$ & Sy 1 & 0.10 & 14.6 & no apparent features \\
$13370-3123^{3}$ & HII & 0.12 & 11.0 & dust lane; (Sersic et al.,1972) \\
$14082+1347$ & Sy 2 & 1.10 & 15.8 & interleaving shells \\
$14164-7236$ & Sy 2 & 1.24 & 16.9 & disturbed, 3 central knots \\
$16343-1023$ & $\ldots$ & $\ldots$ & 19.5 & no apparent features \\
$16380-8120$ & $\ldots$ & $\ldots$ & $\ldots$ & no apparent features \\
$18526-2445$ & $\ldots$ & 1.34 & 17.2 & no apparent features \\
$20253-8152$ & HII & 0.96 & 17.6 & no apparent features \\
& & & & \\
\hline
\end{tabular}

1) Mkn 1239 , 2) MGC-6-30-15 , 3) NGC 5253 


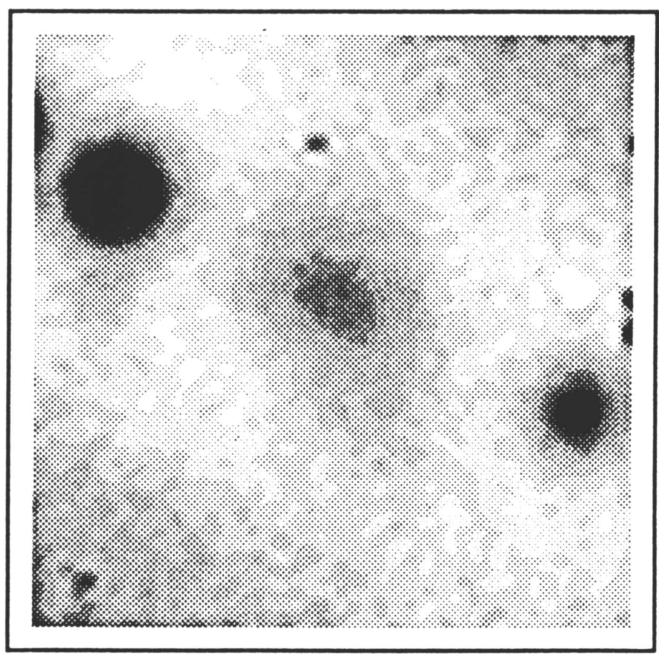

Fig. 1a Unsharp masked R frame $(100 " x 100 ")$ of IRAS 10567.

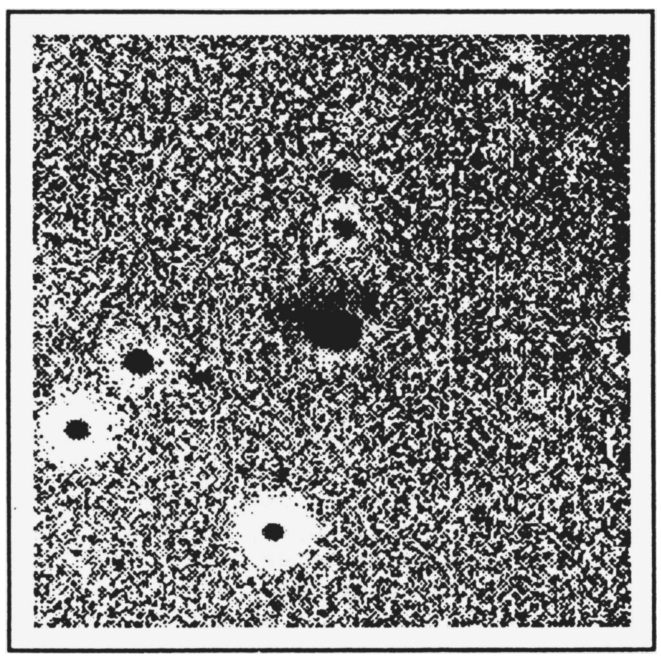

Fig. 2a Unsharp masked B frame (25"x25") of IRAS 14164.

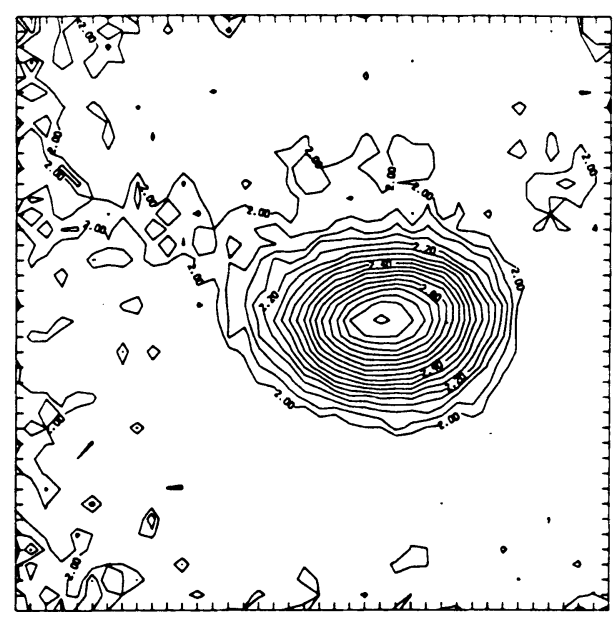

Fig. 1b B-R contour plot of IRAS 10567 ; tickmark interval spacing is 0.5 ".

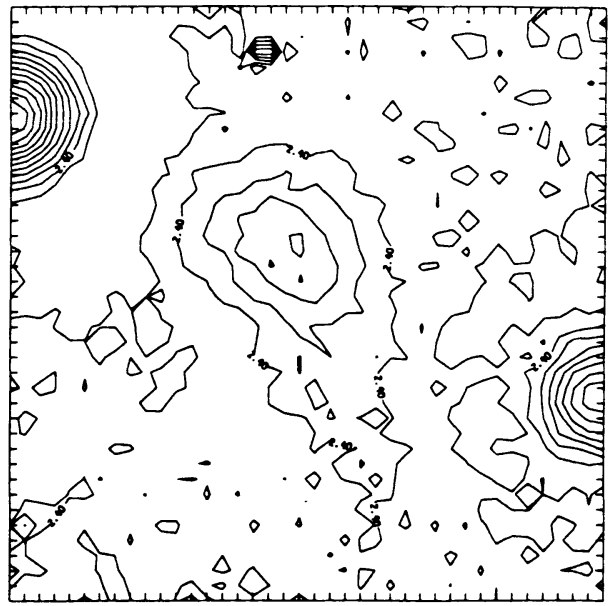

Fig. 2b B-I contour plot of IRAS 14164 ; tickmark interval spacing is $0.125 "$.

\section{REFERENCES}

Schweizer, F. and Ford, W. K., Jr., (1985), in New Aspects of Galaxy Photometry, ed. J-L. Nieto (Berlin:Springer) , p 149

Sersic, J. L., Carranza , G. and Pastoriza , M., (1972), Astrophys. and Space Science, 19, 469

Sparks, W. B., Wall, J. V., Thorne, D. J., Jorden, P. R., van Breda, I. B., Rudd, P. J., and Jorgensen, H. E., (1985), Mon. Not. R. Astron. Soc., 217, 87

Vader, J. P., Frogel, J. A., de Grijp, M. H. K. and Gillett, F. C., (1988); this volume 